\title{
Transverse ultrafast laser inscription in bulk silicon
}

\author{
M. Chambonneau $\odot,{ }^{1,}{ }^{*}$ M. Blothe $\odot,{ }^{1}$ Q. Li $\odot,{ }^{1}$ V. Yu. Fedorov $\odot,{ }^{2,3}$ T. Heuermann,,${ }^{1,4}$ M. Gebhardt $\odot,{ }^{1,4}$ C. Gaida $\odot,{ }^{5}$ \\ S. Tertelmann, ${ }^{6}$ F. Sotier, ${ }^{6}$ J. Limpert, ${ }^{1,4,7}$ S. Tzortzakis, ${ }^{2,8,9}$ and S. Nolte $\oplus^{1,7}$ \\ ${ }^{1}$ Institute of Applied Physics, Abbe Center of Photonics, Friedrich Schiller University Jena, Albert-Einstein-Straße 15, 07745 Jena, Germany \\ ${ }^{2}$ Science Program, Texas A\&M University at Qatar, P.O. Box 23874, Doha, Qatar \\ ${ }^{3}$ P. N. Lebedev Physical Institute of the Russian Academy of Sciences, 53 Leninskiy Prospekt, 119991 Moscow, Russia \\ ${ }^{4}$ Helmholtz-Institute Jena, Fröbelstieg 3, 07743 Jena, Germany \\ ${ }^{5}$ Active Fiber Systems GmbH, Ernst-Ruska-Ring 17, 07745 Jena, Germany \\ ${ }^{6}$ Innolas Photonics GmbH, Justus-von-Liebig-Ring 8, 82152 Krailling, Germany \\ ${ }^{7}$ Fraunhofer Institute for Applied Optics and Precision Engineering IOF, \\ Center of Excellence in Photonics, Albert-Einstein-Straße 7, 07745 Jena, Germany \\ ${ }^{8}$ Institute of Electronic Structure and Laser (IESL), Foundation for Research and Technology-Hellas (FORTH), \\ P.O. Box 1527, GR-71110 Heraklion, Greece \\ ${ }^{9}$ Materials Science and Technology Department, University of Crete, 71003 Heraklion, Greece
}

(Received 30 April 2021; revised 30 July 2021; accepted 9 September 2021; published 14 October 2021)

\begin{abstract}
In-volume ultrafast laser direct writing of silicon is generally limited by strong nonlinear propagation effects preventing the production of modifications. By using advantageous spectral, temporal, and spatial conditions, we demonstrate that modifications can be repeatably produced inside silicon. Our approach relies on irradiation at $\approx 2 \mu \mathrm{m}$ wavelength with temporally distorted femtosecond pulses. These pulses are focused in a way that spherical aberrations of different origins mutually balance, as predicted by point spread function analyses and in good agreement with nonlinear propagation simulations. We also establish the laws governing modification growth on a pulse-to-pulse basis, which allows us to demonstrate transverse inscription inside silicon with various line morphologies depending on the irradiation conditions. We finally show that the production of single-pulse repeatable modifications is a necessary condition for reliable transverse inscription inside silicon.
\end{abstract}

DOI: 10.1103/PhysRevResearch.3.043037

\section{INTRODUCTION}

Ultrafast laser direct writing is a proven technique for involume functionalization of dielectrics in a three-dimensional (3D), fast, and contactless way [1]. Despite its high desirability for applications including photonics, sensors, photovoltaics, ultrafast microelectronics, and quantum computing, this technique has no equivalent in monolithic silicon [2]. This is because filaments are formed as ultrashort laser pulses propagate in this highly nonlinear material [3-8]. As a consequence of these nonlinear propagation effects in silicon, the energy deposition is strongly delocalized to the prefocal region at levels below the damage threshold - thus representing an uttermost challenge for the production of repeatable modifications which are the building blocks for 3D laser direct writing. While different approaches have been devised for writing lines longitudinally (i.e., along the optical axis) in silicon [9-15], there are few demonstrations of transverse line

\footnotetext{
*maxime.chambonneau@hotmail.fr
}

Published by the American Physical Society under the terms of the Creative Commons Attribution 4.0 International license. Further distribution of this work must maintain attribution to the author(s) and the published article's title, journal citation, and DOI. inscription (i.e., perpendicular to the optical axis), especially in the femtosecond regime. Two strategies have been adopted so far for solving this issue. The first one consists of using longer pulses (i.e., in the picosecond or nanosecond regime) to decrease nonlinearities [16-22]. The main disadvantage of this approach lies in heating, which limits the precision that can be achieved on the inscribed structures. In order to reduce the heat-affected zone, a second strategy consists of employing femtosecond pulses with an altered entrance surface, either by depositing an oxide layer [23] or by employing oil immersion [24]. In this case, the oxide layer or the index-matching fluid often has to be removed after the laser inscription. In addition to this impractical aspect, surface alteration is undesirable - if not impossible — in numerous applications.

In this paper, we demonstrate contactless femtosecond laser transverse inscription inside silicon by employing advantageous spectral, temporal, and spatial conditions. We use femtosecond laser pulses at $\approx 2 \mu \mathrm{m}$ wavelength, where the peak in the nonlinear refractive index gives rise to enhanced energy deposition as shown in Refs. [5,22,25]. In the time domain, our procedure relies on the utilization of temporally distorted ultrashort pulses. Temporal imperfections were recently shown to act as a seed for creating permanent modifications inside silicon [26,27]. In the spatial domain we determine the focus position for which the 
spherical aberration induced by the focusing lens and the one created by the refractive index mismatch at the air-silicon interface mutually balance, as supported by point spread function calculations. This approach allows us to demonstrate the production of repeatable permanent internal modifications with ultrashort laser pulses, in good agreement with nonlinear propagation simulations. The laws governing modification growth after successive irradiations at the same location are then established. This understanding of the damage growth behavior leads us to devise transverse femtosecond laser inscription of continuous lines in the bulk of silicon. Three distinct line morphologies which depend on the writing speed and the input pulse energy are exhibited. Ultimately, we demonstrate that continuous lines can only be written when the single-pulse modification probability is $100 \%$-thus shedding light on the necessity to reignite damage on a pulseto-pulse basis during the inscription.

\section{EXPERIMENTAL ARRANGEMENT}

The experimental arrangement employed for inducing, detecting, and characterizing permanent modifications inside silicon is depicted in Fig. 1(a). It relies on 750-fs-duration pulses [full width at half maximum, assuming a squared hyperbolic secant $\left(\mathrm{sech}^{2}\right)$ temporal shape] at $1.95 \mu \mathrm{m}$ center wavelength emitted at $\Omega_{0}=100-\mathrm{Hz}$ repetition rate by a Tm-doped fiber laser source. The spectral width is $\approx 20 \mathrm{~nm}$, ensuring that chromatic aberration is negligible in the experiments. The input pulse energy $E_{\text {in }}$ is controlled by means of a half-wave plate and a linear polarizer. This energy is measured in air, between the focusing optics and the sample (i.e., the Fresnel reflections are not taken into account), and it may reach $E_{\text {in }} \approx 2.55 \mu \mathrm{J}$. The beam size before focusing is adjusted by means of a Galilean telescope so that the beam diameter is larger than the diameter of the asphere [Thorlabs, C037TME-D, numerical aperture $(\mathrm{NA}=0.85)$ ] employed for focusing the beam inside the silicon. This lens can be displaced along the optical axis $z$. Since the asphere used is designed for operation at a wavelength of $9.5 \mu \mathrm{m}$, there are spherical aberrations at the laser wavelength used here (i.e., spherochromatism [28,29]) in addition to aberrations due to the index mismatch at the air-silicon interface (see Sec. III A) $[28,30]$. The silicon sample (Siegert Wafer, double-side polished, $1 \mathrm{~mm}$ thickness, $\langle 100\rangle$ oriented, undoped, $200 \Omega \mathrm{cm}$ resistivity) can be moved in the $x y$ plane perpendicular to the optical axis $z$ thanks to a positioning system (Aerotech, ANT 130).

Three-dimensional in situ monitoring of the laser-silicon interaction is performed by means of two customized diagnostics. The first one is a transmission microscope relying on white light illumination. The light passes through the sample along the $x$ axis, and images of the modifications in the $y z$ plane are recorded thanks to an objective lens (Mitutoyo, M Plan Apo NIR-HR 50×), a tube lens, and an InGaAs camera (Xenics, Bobcat 320). Images in the $x y$ plane are recorded by a transmission microscope relying on illumination at $1.03 \mu \mathrm{m}$ (Spectra-Physics, femtoTrain) along the $-z$ direction passing through the silicon sample, the focusing asphere, and a tube lens before being collected by a silicon-based camera. This latter microscope is utilized for determining the relative
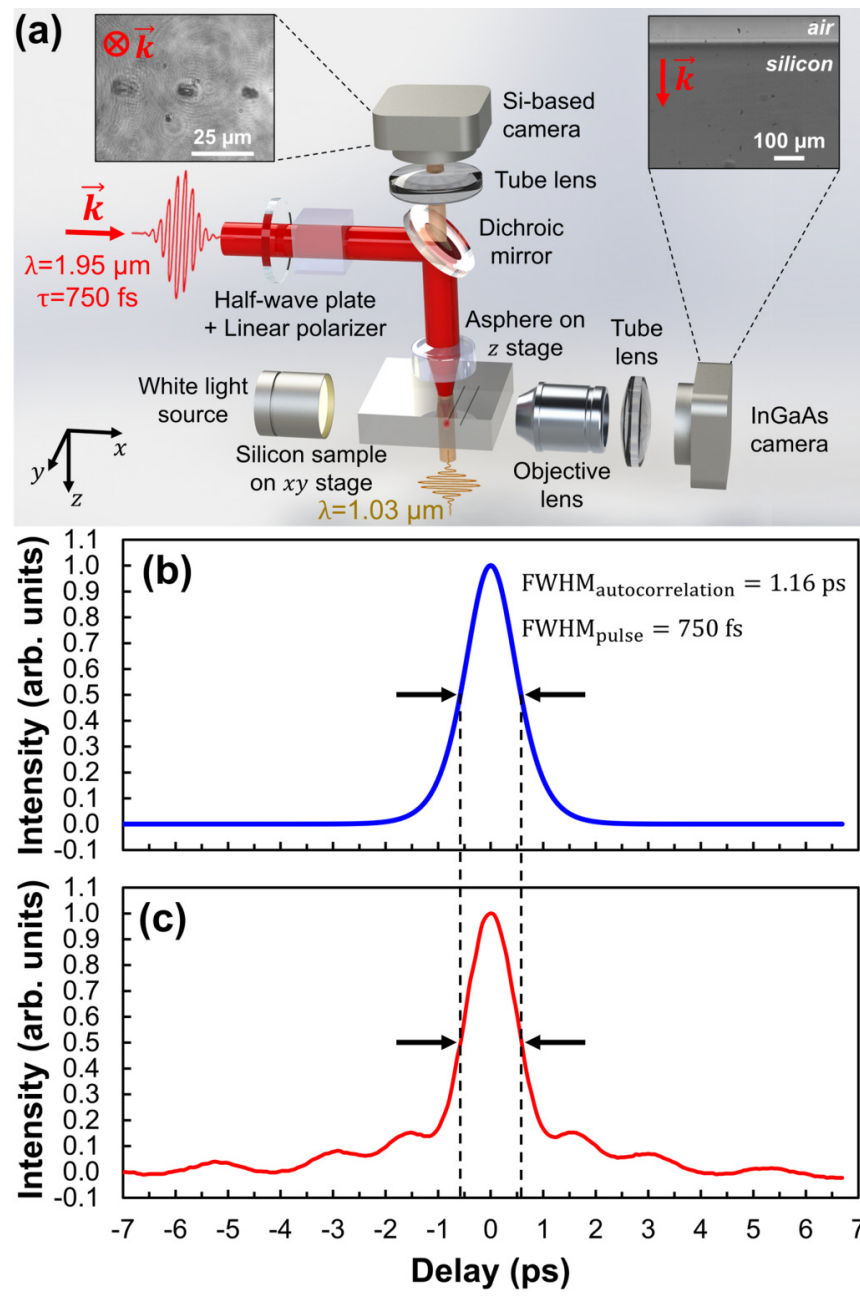

FIG. 1. (a) Schematic of the arrangement employed for internal damage and transverse line writing in silicon and associated in situ diagnostics. The vector $\vec{k}$ indicates the direction of laser propagation. The second-order autocorrelation traces in (b) and (c) have been obtained with the two distinct $1.95-\mu \mathrm{m}$ laser sources utilized in our study.

position of the focus with respect to the sample surface by performing surface damage scans at near-threshold energies. The hypothetical focus position is obtained by multiplying the displacement $\Delta z$ of the focusing lens along the optical axis (starting from the entrance surface) by the refractive index of silicon at $1.95 \mu \mathrm{m}\left(n_{0} \approx 3.45[31]\right)$. For improved imaging performance, ex situ characterizations of the modifications are carried out with a transmission infrared phase microscope consisting of a customized Mach-Zehnder interferometer operated at $1.3 \mu \mathrm{m}$ wavelength, the principles of which are detailed in Ref. [32].

Two laser sources with similar spectral and spatial features are used. However, the temporal shapes of the pulses emitted by these laser systems are different. The first laser system (Active Fiber Systems; see details in Ref. [33]) delivers undistorted bell-shaped pulses as illustrated in Fig. 1(b). In contrast, as shown by the second-order autocorrelation trace in Fig. 1(c), the femtosecond pulses emitted by the second laser source (InnoLas Photonics, FEMTO*1950-8-T-2500) exhibit 
a structured temporal profile. This distortion originates from uncompensated higher-order dispersion caused by a mismatch between the stretcher and the compressor and results in the existence of satellite peaks. However, one has to emphasize that second-order autocorrelation does not allow one to discriminate whether these peaks are prepulses or postpulses.

\section{RESULTS AND DISCUSSION}

\section{A. Linear propagation calculations}

Let us first examine the focus position that allows us to maximize the energy deposition inside silicon. To do so, we have first carried out linear propagation calculations in silicon with our recently developed in-house vectorial model called INFOCUS $[34,35]$. Briefly, this model gives access to the linear propagation in any medium and relies on point spread function analysis, allowing the determination of the volumetric field distribution of light in the focal region. One specificity of this model is that it accounts not only for the spherical aberration created by the refractive index mismatch, but also for the one induced by the focusing lens through its associated Zernike polynomials. While this latter spherical aberration may be neglected for a beam focused with an objective lens, it becomes significant for thick singlet lenses with short focal length. The accuracy of our model to predict these two types of spherical aberrations separately was benchmarked with propagation imaging [34]. While, in principle, similar measurements could be performed in the present study, these would require an objective lens with a numerical aperture higher than that of the focusing asphere (i.e., NA > 0.85), which represents a major technical challenge. In this paper, we thus restrict ourselves to linear propagation calculations. As mentioned in Sec. II, because of the difference between the design wavelength of this asphere $(9.5 \mu \mathrm{m})$ and the laser wavelength $(1.95 \mu \mathrm{m})$, spherical aberration is inevitably induced by the focusing lens.

For simplicity, let us first consider that the propagation is linear, i.e., nonlinear effects such as the optical Kerr effect as well as plasma formation and defocusing are neglected. Under this assumption, the results are independent of the temporal pulse shape-and thus are valid for the two employed laser systems [see Figs. 1(b) and 1(c)]. Figure 2(a) shows intensity distributions for various focus positions in silicon. For low focus positions $(\leqslant 80 \mu \mathrm{m})$, the asymmetry in the intensity distributions is ascribable to the predominance of lens-induced spherical aberration. Conversely, for deep focus positions $(\geqslant 140 \mu \mathrm{m})$, elongated patterns along the propagation direction indicate the predominance of spherical aberration created by the refractive index mismatch at the air-silicon interface. Interestingly, for intermediate positions $(\approx 100 \mu \mathrm{m})$, both types of spherical aberrations mutually balance, leading to localized near-symmetric intensity distributions. This is further confirmed in Fig. 2(b), where the intensity is the highest for the $100-\mu \mathrm{m}$ focus position. This leads us to the conclusion that balancing the two types of spherical aberrations is beneficial for creating damage inside silicon. However, one should emphasize that this condition on mutually balancing spherical aberrations taken alone cannot fully predict damage experiments as the hypothesis of a linear propagation is unrealistic given our experimental (a)
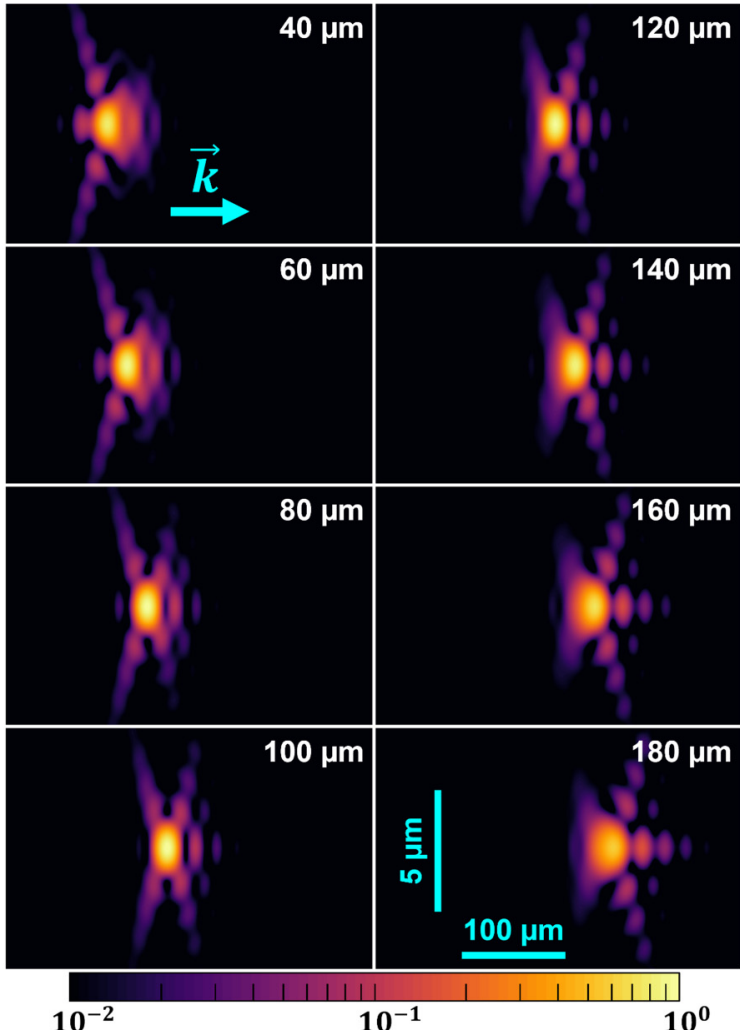

(b)
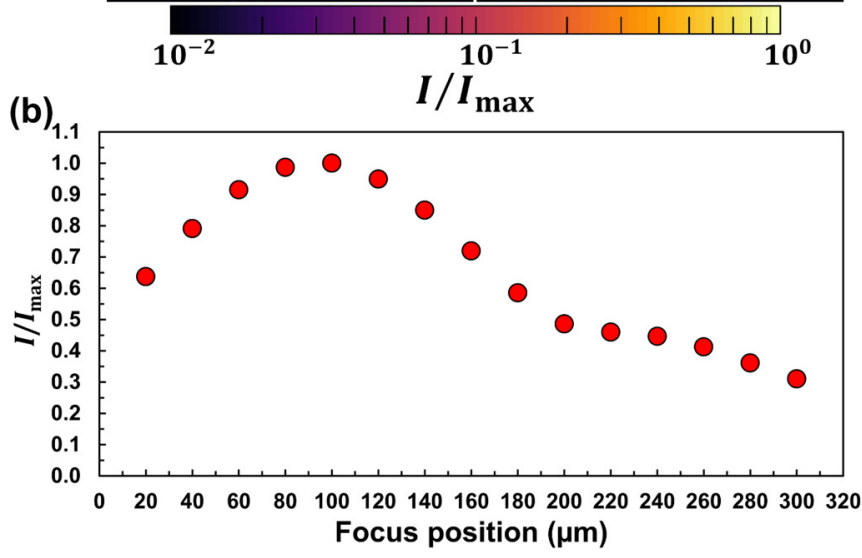

FIG. 2. (a) Numerical calculations of the on-axis intensity distribution for various focus positions thanks to our linear propagation model [34,35]. The vector $\vec{k}$ indicates the direction of laser propagation. The intensity distributions $I / I_{\max }$ are normalized to the maximum intensity of the whole set of data. The spatial scale applies to all images. (b) Evolution of the normalized maximum intensity for each configuration as a function of the focus position.

conditions. Indeed, the nonlinear refractive index of silicon at $\lambda=1.95 \mu \mathrm{m}$ wavelength is $n_{2} \approx 9.4 \times 10^{-18} \mathrm{~m}^{2} / \mathrm{W}$ [36-38]. The corresponding critical power for self-focusing $P_{\mathrm{cr}} \approx 1.8962 \lambda^{2} /\left(4 \pi n_{0} n_{2}\right) \approx 17.7 \mathrm{~kW}$ is much lower than the experimental peak powers $(P \geqslant 1.7 \mathrm{MW})$. Therefore, while spherical aberrations are found experimentally as a linear effect, nonlinear propagation effects need to be considered to determine the optimal focus position.

\section{B. Nonlinear propagation simulations}

In order to consider the nonlinear propagation inside silicon, we performed numerical simulations based on the 
unidirectional pulse propagation equation (UPPE) $[6,39,40]$. As the initial condition at the entrance of the silicon sample, we took a pulse with the spatial profile calculated by our vectorial model, which allowed us to include the effect of aberrations in the simulations. The considered temporal shape corresponds to the autocorrelation trace in Fig. 1(b). The input pulse energy was chosen to be $1 \mu \mathrm{J}$. Together with the UPPE, we solved the following equation for the electron density $\rho(x, y, z, t)[5,6,41]$ :

$$
\frac{\partial \rho}{\partial t}=\sigma_{2} I^{2}\left(\rho_{\mathrm{nt}}-\rho\right)+\sigma_{\mathrm{B}} \frac{\mathcal{E}^{2}}{U_{\mathrm{cr}}} \frac{\rho_{\mathrm{nt}}-\rho}{\rho_{\mathrm{nt}}} \rho-\gamma \rho^{3}-\frac{\rho}{\tau_{\mathrm{r}}} .
$$

Equation (1) is a standard plasma rate equation used for simulations of laser pulse propagation in gases and dielectrics [41]. Recently, this kind of simulation was applied for semiconductors represented by silicon $[5,6]$. The first term on the right-hand side describes two-photon ionization, the second term describes avalanche ionization, the third term describes Auger recombination, and the fourth term describes electron relaxation. Here, $\rho_{\mathrm{nt}}=5 \times 10^{28} \mathrm{~m}^{-3}$ is the density of neutral atoms, $\sigma_{2}=\beta_{2} /\left(2 \hbar \omega_{0} \rho_{\mathrm{nt}}\right)$ is the cross section of two-photon ionization with the two-photon absorption coefficient $\beta_{2}=$ $2.97 \times 10^{-12} \mathrm{~m} / \mathrm{W}[36,37]$, and $I(x, y, z, t)$ is the pulse intensity; $\sigma_{\mathrm{B}}=\left(e^{2} / m_{e}^{*}\right) v_{c} /\left(v_{c}^{2}+\omega_{0}^{2}\right)$ is the inverse bremsstrahlung cross section, where $e, m_{e}$, and $m_{e}^{*}=0.5 m_{e}$ are the elementary charge, the electron mass, and the effective electron mass, $v_{c}=0.3 \times 10^{15} \mathrm{~s}^{-1}$ is the collision frequency [42], $\omega_{0}$ is the central pulse frequency, and $\mathcal{E}(x, y, z, t)$ is the pulse electric field, while $U_{\mathrm{cr}}=1.5 U_{\mathrm{i}}$ with $U_{\mathrm{i}}=1.12 \mathrm{eV}$ being the bandgap energy (the coefficient 1.5 accounts for the momentum conservation); $\gamma=1.1 \times 10^{-42} \mathrm{~m}^{6} / \mathrm{s}$ is the Auger recombination coefficient [43], and finally, $\tau_{\mathrm{r}}=100 \mathrm{fs}$ is the electron relaxation time, which we took from Ref. [44] assuming that, due to collisions, the maximum energy of free electrons cannot be much larger than the band gap. The last term in Eq. (1) allows us to account in a phenomenological manner for such crucial effects of electron interaction with the lattice as electron-phonon scattering and electron-defect interactions. Ultimately, free-carrier diffusion is neglected as this mechanism is not expected to play a major role at the time scale of ultrashort pulses [45].

Figure 3 summarizes our simulation results. Figure 3(a) shows the dependence of the peak fluence on the propagation distance for different focus positions. Here, one can note that the maximum fluence values are almost independent of the focus position. Therefore, based on the fluence data, we cannot conclude that some focus positions are preferable to others. In turn, Fig. 3(b) shows the dependence of the peak intensity $I_{\text {peak }}$ and the peak electron density $\rho_{\text {peak }}$ on the focus position, both exhibiting a radically different behavior compared with the fluence profiles in Fig. 3(a). This difference originates from the nonlinear dynamics (including self-phase modulation, Kerr effect, and plasma nonlinearities) in the interaction zone. The values of $I_{\text {peak }}$ and $\rho_{\text {peak }}$ reach their respective maxima at $100-\mu \mathrm{m}$ focus position, in accordance with the predictions made by our vectorial model (see Fig. 2). However, since the intensity and electron density reach their peak values at some arbitrary point in space (across the beam) and time, one cannot fully rely on these data. Moreover, it
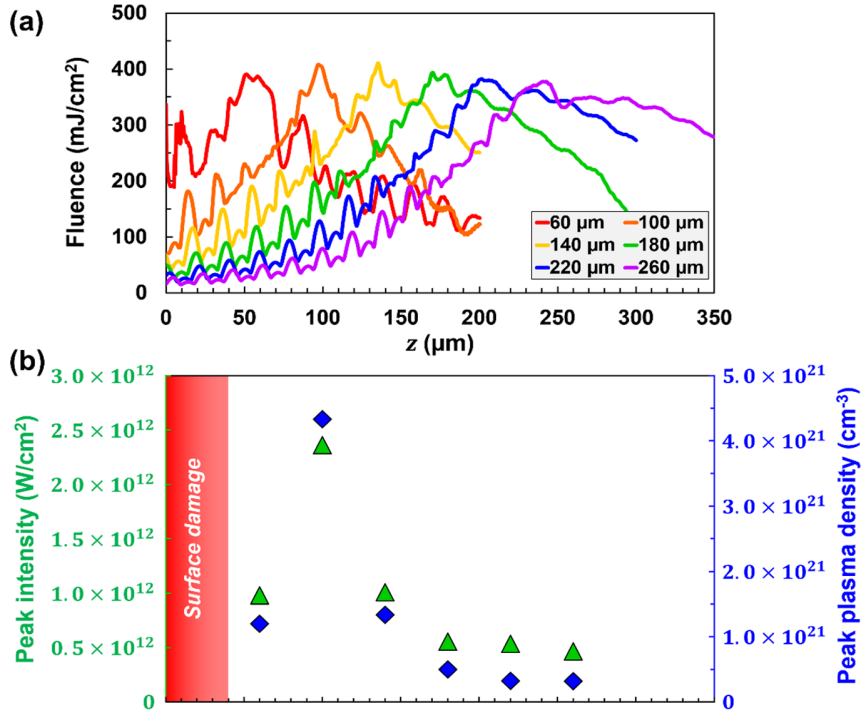

(c)

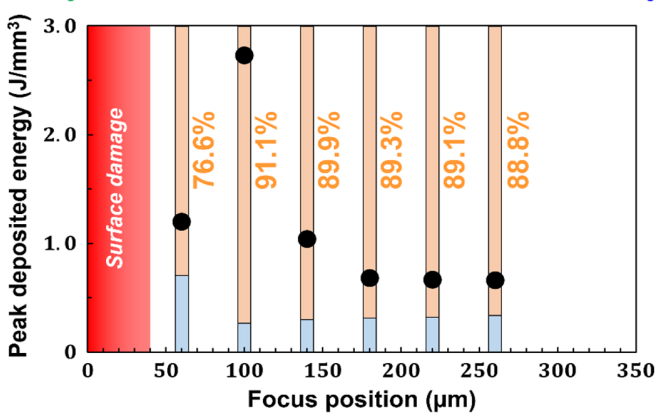

FIG. 3. Results of numerical simulations of nonlinear propagation in silicon. (a) On-axis fluence profiles for different focus positions. (b) Evolution of the peak intensity (green) and peak electron density (blue) as a function of the focus position. The red region indicates the focus positions for which surface damage was experimentally detected. (c) Evolution of the peak deposited energy as a function of the focus position (black circles). The bars indicate the percentage of the input laser energy spent on ionization (blue) and acceleration (i.e., heating) of electrons (orange, with the indicated percent value).

is known that criteria based on the peak electron density usually do not provide the most accurate description of damage experiments [46]. Therefore, as a more reliable damage criterion, we decided to calculate the laser energy deposited to the free-electron subsystem, which then can be transferred to the crystal lattice [47] (see the details in Appendix A). The peak values of the deposited energy (estimated by neglecting surface damage for focus positions $\leqslant 40 \mu \mathrm{m}$ ) are displayed in Fig. 3(c) as a function of the focus position. The peak deposited energy is the highest for the $100-\mu \mathrm{m}$ focus position, indicating that damage should be preferentially produced at this position.

Additionally, since in our nonlinear propagation model the laser energy is only spent on ionization and electron acceleration (i.e., heating), by calculating the total laser energy loss and the total energy deposited to free electrons, we can separately find the percent of the initial laser energy spent on ionization and electron heating [see the color bars in Fig. 3(c)]. In particular, we find that not only the peak 


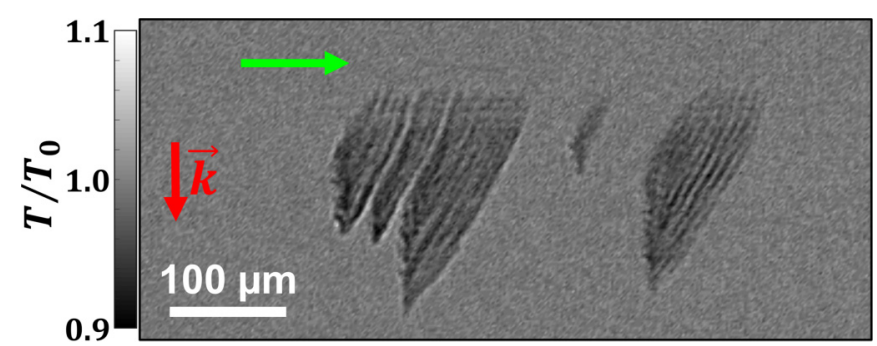

FIG. 4. Transmission $\left(T / T_{0}\right)$ image of transverse line inscription attempt with pulses corresponding to the autocorrelation trace in Fig. 1(b) at the writing speed $v=20 \mu \mathrm{m} / \mathrm{s}$. The vector $\vec{k}$ and the green arrow indicate the direction of laser propagation and the writing direction, respectively.

deposited energy, but also the percent of laser energy spent on electron acceleration is the highest $(>90 \%)$ for the $100-\mu \mathrm{m}$ focus position.

To sum up, both the linear and the nonlinear propagation simulations indicate an optimal focus position near $100 \mu \mathrm{m}$, which could thus potentially lead to continuous transverse line writing inside silicon. In order to verify experimentally these theoretical predictions, we have carried out damage experiments in the bulk of silicon using the laser source emitting temporally undistorted pulses [see Fig. 1(b)]. These experiments consist of single-pulse irradiation, and the corresponding binary result (i.e., damage produced or not) is evaluated by means of the three-dimensional in situ monitoring system described in Sec. II. By repeating the experiments on ten different fresh sites, the bulk modification probability (i.e., the ratio between the number of damage sites and the total tested sites) is evaluated, and it lies in the $0-30 \%$ range. In other words, no repeatable modification could be produced in the bulk of silicon. This observation held even by changing the input pulse energy as well as the focus position. As illustrated by the attempted transverse line inscription in Fig. 4, where modifications are randomly produced during writing, transverse inscription of continuous lines in this stochastic regime is doomed to failure. Both the size and the depth of the modifications are unrepeatable. This random behavior for damage production cannot originate from fluctuations of the laser power due to the high stability $(\approx 1 \%)$ of the employed system. It is thus more likely related to the existence of precursor defects inside the material [48]. One may note the limited spatial expansion of the modifications along the writing direction (indicated by the green arrow) in Fig. 4 . The modifications can only be propagated over $\approx 100 \mu \mathrm{m}$ before no more expansion occurs.

The negative results in Fig. 4 obtained with beneficial spectral and spatial conditions lead us to improve the temporal pulse shape. In the nanosecond regime, several studies have shown that the temporal pulse shape strongly influences the production [49-53] and the morphology [54,55] of laserproduced modifications in fused silica. Recent results showed that the temporal pulse shape is also a key parameter in femtosecond-laser-silicon interaction as a perfect bell-shaped temporal profile is not suitable for damage production inside silicon [26]. Hereafter, we employ temporally distorted pulses associated with the autocorrelation trace in Fig. 1(c).

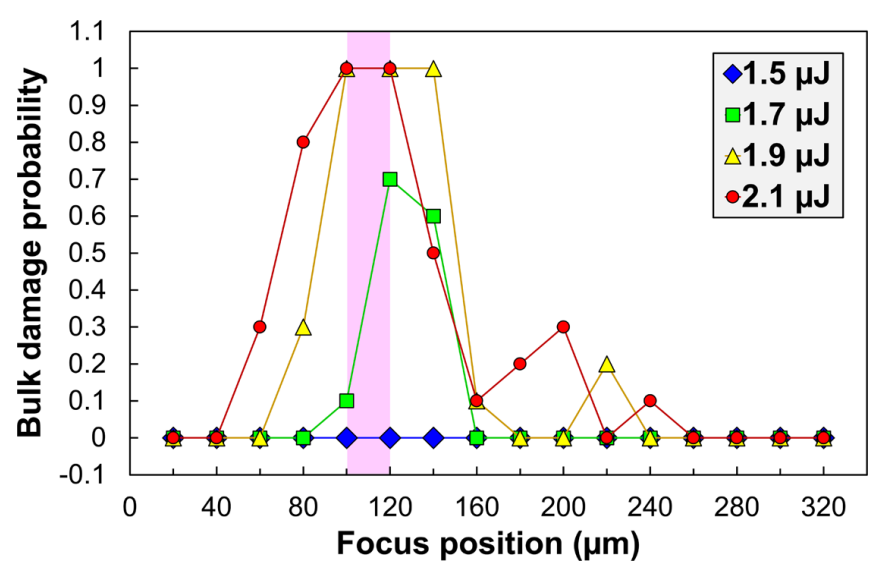

FIG. 5. Evolution of the bulk damage probability in silicon with single laser pulses as a function of the focus position for various $E_{\mathrm{in}}$. The pink region indicates the positions for which damage probability reaches $100 \%$ for $E_{\text {in }} \geqslant 1.9 \mu \mathrm{J}$.

\section{Single-pulse damage production}

In order to investigate the possibility offered by our procedure for inducing internal modifications in silicon, single-pulse damage production experiments have been first realized. The corresponding results are shown in Fig. 5 in terms of bulk damage probability as a function of the focus position for various input pulse energies $E_{\text {in }}$. Surface damage was systematically detected for focus positions $\leqslant 40 \mu \mathrm{m}$ (not represented in Fig. 5). No bulk damage is observed for any focus position at $E_{\text {in }}=1.5 \mu \mathrm{J}$, thus defining the modification threshold. The striking feature in Fig. 5 is the existence of a narrow window (indicated in pink) for $100-120-\mu \mathrm{m}$ focus position, where the damage probability shows a maximum value for $E_{\text {in }} \geqslant 1.7 \mu \mathrm{J}$, in excellent agreement with the theoretical predictions in Secs. III A and III B. Therefore this narrow window indicates the focus position for which spherical aberrations induced by the lens and the air-silicon interface mutually balance. Interestingly, a bulk damage probability of $100 \%$ is reached for $E_{\text {in }} \geqslant 1.9 \mu \mathrm{J}$. This deterministic character suggests that these conditions are well adapted for ultrafast laser direct writing inside silicon, in opposition to the previous results obtained with temporally undistorted pulses (Fig. 4). Apart from this narrow window, nonzero modification probabilities $<100 \%$ are measured, suggesting nonintrinsic damage likely originating from the existence of local precursor defects [48] - thus incompatible with the repeatable inscription of functions inside silicon (see Fig. 4).

Besides the statistical aspects related to ultrafast laserinduced damage inside silicon, we have investigated the associated material changes. To do so, the morphology of single-pulse modifications has been characterized with different methods. First, in situ transverse observations reveal that the modifications consist of multiple microbubbles along the optical axis $z$, as shown in Fig. 6(a). The number of individual bubbles varies from one to four in the tested input energy range, and their typical size is $\approx 4 \mu \mathrm{m}$. Assuming that the deepest bubble is formed first, this damage morphology can be interpreted thanks to the moving breakdown model [56], which implies that the produced plasma moves toward the 


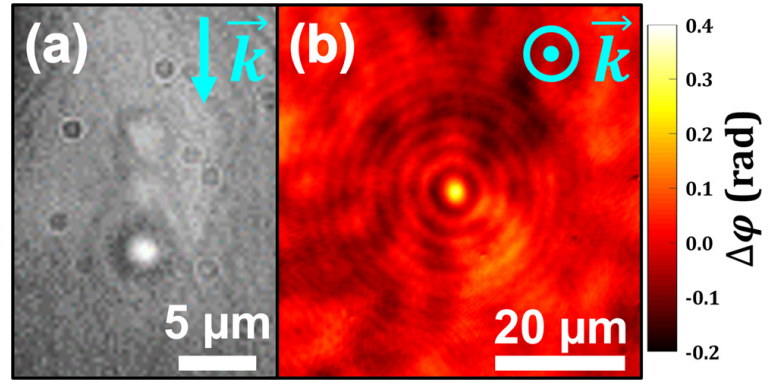

FIG. 6. Morphology of a single-pulse modification produced at $E_{\text {in }}=2.1 \mu \mathrm{J}$. (a) In situ transmission microscopy image of the modification in the $y z$ plane. (b) Ex situ phase image of the modification in the $x y$ plane. The phase shift $\Delta \varphi$ is mapped out thanks to a four-step procedure described in Ref. [32]. The concentric circles are artifacts originating from diffraction. The vector $\vec{k}$ indicates the direction of laser propagation.

incoming beam during the laser pulse. Ex situ phase measurements have also been carried out in the $x y$ plane, as shown in Fig. 6(b). The phase shift—and thus the refractive index change-associated with the laser-produced modifications is positive. Two main types of material change may explain such an observation. First, the positive refractive index change may originate from local laser-induced strain inside the material, analogously to damage produced in the nanosecond and picosecond regimes [12,13,19,57]. Second, the modifications may consist of an allotropic change in silicon, from monocrystalline to polycrystalline [58], or other polymorphs [59].

\section{Multipulse modification growth}

Establishing the laws governing modification growth (i.e., the increase in the modification size on a pulse-to-pulse basis) in silicon is a prerequisite for envisioning multipulse functionalization processes. While, previously, we have determined the conditions leading to repeatable single-pulse permanent modifications (see Fig. 5), experiments on the evolution of the modification size after successive irradiations have been carried out. Such a growth sequence is illustrated by transmission microscopy images in the $x z$ plane after different numbers of applied pulses in Fig. 7(a). The first pulse at $120-\mu \mathrm{m}$ focus position initiates a modification similar to the one in Fig. 6. This barely detectable single-shot modification expands spatially after several irradiations to form a strongly absorbing damage site. Two processes may explain this growth behavior. First, damage created by one pulse can act as a precursor defect which seeds the plasma production during the subsequent pulse through electric field enhancement [48]. Second, the positive refractive index change in Fig. 6(b) can be attributed to strain, which may decrease the band gap of silicon locally $[60,61]$ - and thus increase the absorption. One may note the formation of a tail growing rapidly toward the entrance surface for a number of pulses $N \geqslant 30$, as indicated by white arrows in Fig. 7(a). While deeper investigations out of the scope of the present study are necessary for establishing the physical origin of this tail, it must be emphasized that its expansion does not create any detectable surface damage. Quantitative analyses of the evolution of the modification lengths $L_{x}$ and $L_{z}$
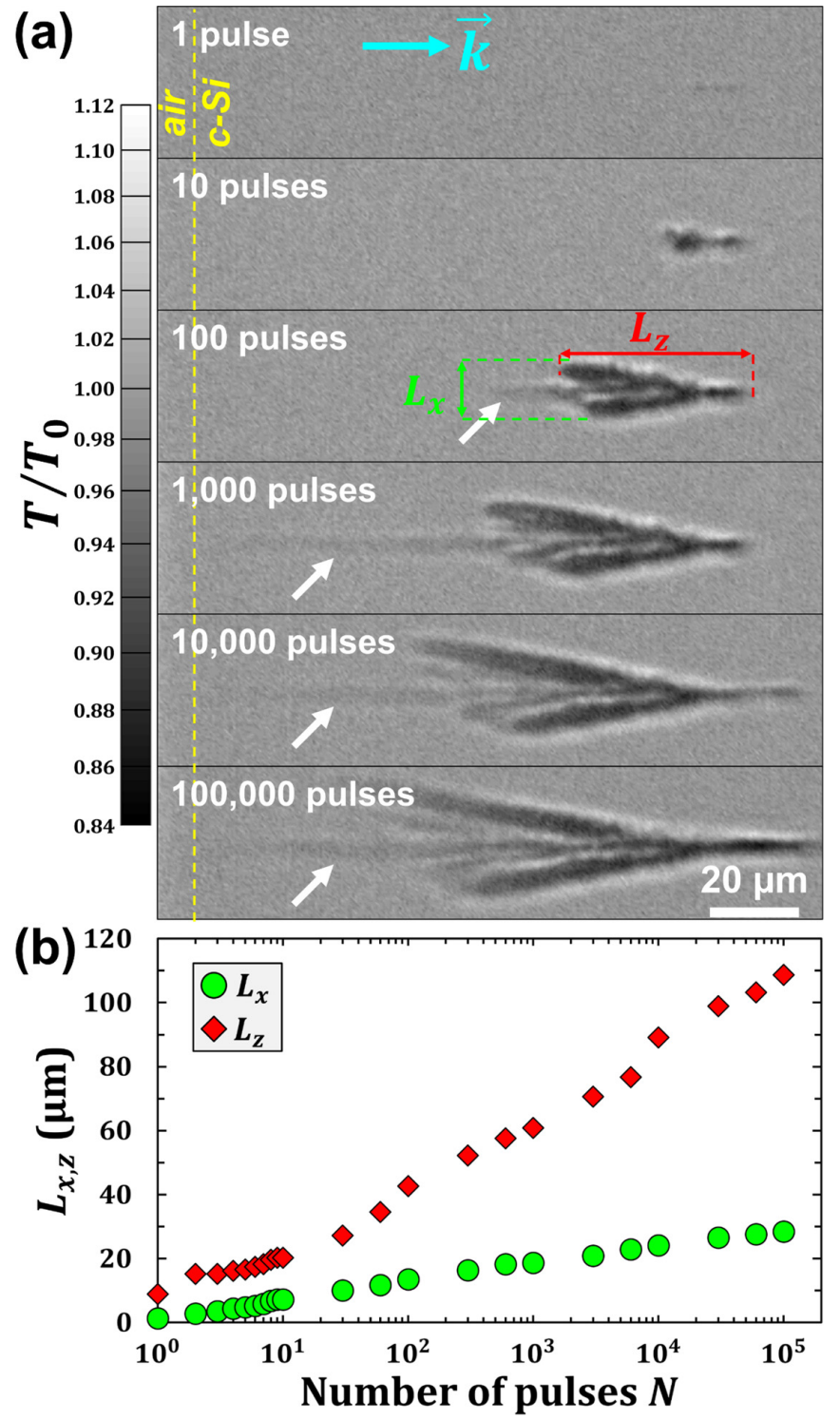

FIG. 7. Laser-induced modification growth sequence inside silicon for $E_{\text {in }}=2.1 \mu \mathrm{J}$. (a) Transmission $\left(T / T_{0}\right)$ images of a modification for different numbers of irradiations at the same focus position $(120 \mu \mathrm{m})$. The spatial scale applies to all images. The vector $\vec{k}$ indicates the direction of laser propagation. The dashed yellow line indicates the sample entrance surface. (b) Evolution of the modification length $L_{x}$ and $L_{z}$ indicated in (a) along the $x$ and the $z$ axis, respectively, as a function of the number of applied pulses $N$.

indicated in Fig. 7(a) along the $x$ and the $z$ axis, respectively, are shown in Fig. 7(b) as a function of the number of pulses $N$. Both $L_{x}$ and $L_{z}$ scale logarithmically with the number of pulses $N$. Remarkably, this evolution for bulk modifications is slower than for surface modifications in fused silica, which usually exhibit linear and exponential growth on the entrance and the exit surface, respectively [62-64].

Additional growth experiments have been carried out for different input pulse energies. The corresponding results are displayed in Fig. 8. The evolution of the modification lengths $L_{x, y}$ and $L_{z}$ is shown in Figs. 8(a) and 8(b), respectively, as a function of the number of pulses $N$. Similar to the trends in 

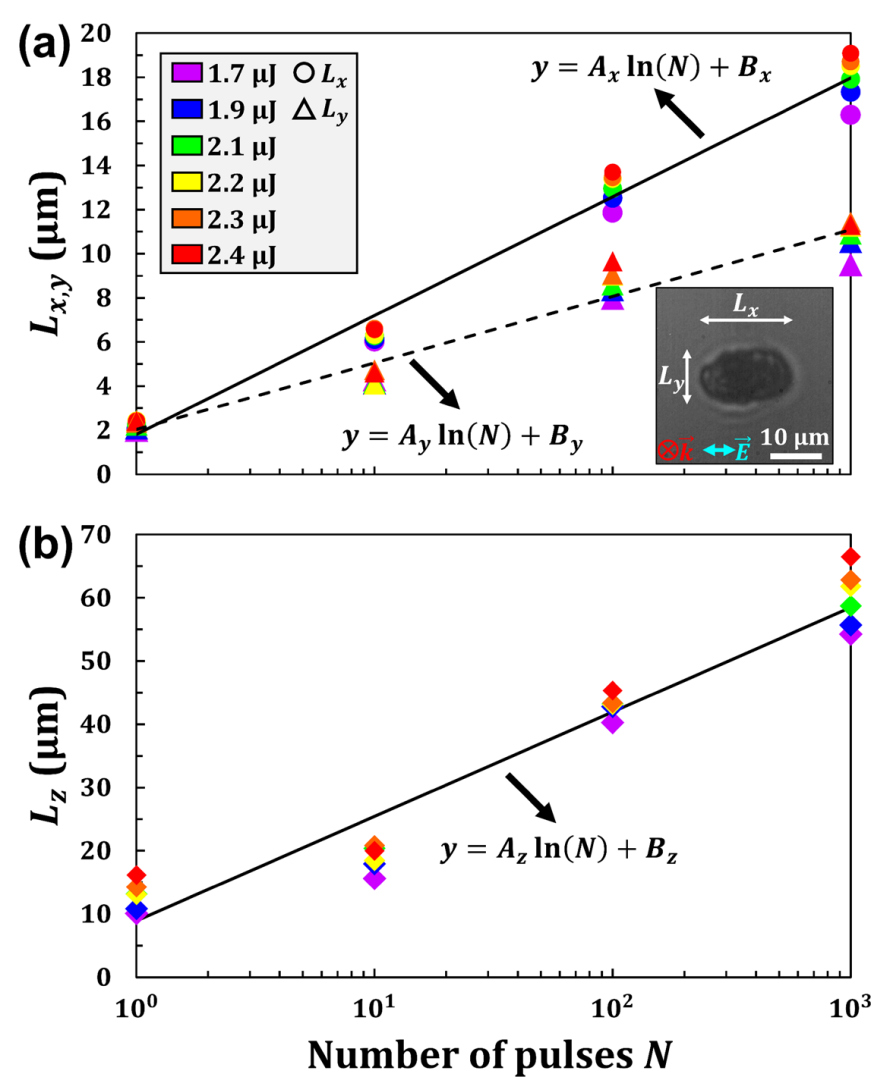

FIG. 8. Laws of modification growth inside silicon. (a) Evolution of the transverse modification dimensions $L_{x}$ (circles) and $L_{y}$ (triangles) along the $x$ and the $y$ axis, respectively, as a function of the number of applied pulses $N$ for different input pulse energies. The inset is an optical microscopy observation of a modification produced after 1000 irradiations at $E_{\text {in }}=2.4 \mu \mathrm{J}$. The vectors $\vec{k}$ and $\vec{E}$ indicate the direction of laser propagation and the polarization, respectively. (b) Evolution of the modification dimension $L_{z}$ along the optical axis $z$ for the same input pulse energies as in (a). In both (a) and (b), the curves with indicated equations are logarithmic fits of the experimental data according to Eq. (2). The values of the coefficients $A_{i}$ and $B_{i}(i=x, y$, or $z)$ are reported in Table I.

Fig. 7(b), each data set is fairly well fitted by a logarithmic function as

$$
L_{i}=A_{i} \ln (N)+B_{i},
$$

where $i=x, y$, or $z$ and $A_{i}$ and $B_{i}$ are the growth coefficient and the single-pulse modification length along $i$, respectively.

TABLE I. Statistical analysis of the $A_{i}$ and $B_{i}$ coefficients $(i=x$, $y$, or $z$ ) appearing in the laws of modification growth described in Eq. (2). The data have been obtained by analyzing 288 modifications initiated at $120-\mu \mathrm{m}$ focus position by $1,10,100$, and 1000 laser pulses with $E_{\text {in }}=1.7-2.4 \mu \mathrm{J}$. Values are given in micrometers.

\begin{tabular}{lcccccc}
\hline \hline & $A_{x}$ & $B_{x}$ & $A_{y}$ & $B_{y}$ & $A_{z}$ & $B_{z}$ \\
\hline Average & 2.34 & 1.81 & 1.31 & 2.03 & 7.18 & 8.91 \\
Standard deviation & 0.12 & 0.07 & 0.08 & 0.13 & 0.31 & 1.43 \\
Minimum & 2.12 & 1.71 & 1.14 & 1.86 & 6.82 & 6.49 \\
Maximum & 2.48 & 1.90 & 1.38 & 2.25 & 7.66 & 10.54 \\
\hline \hline
\end{tabular}

Given that the characterizations in Fig. 6 have revealed symmetric modifications in the $x y$ plane, one could intuitively expect a conservation of this symmetry in the multipulse configuration - and thus $A_{x}=A_{y}$. As shown in Fig. 8(a), this is not the case, and the modifications are more elongated along $x$ than along $y$. Consecutive irradiations thus produce elliptical modifications whose major and minor axes are parallel and perpendicular to the laser polarization, respectively, as illustrated by the inset in Fig. 8(a). Interestingly, elliptical modifications were also observed in silicon for multipulse irradiations in the bulk $[25,26,65,66]$, as well as for femtosecond irradiations on the exit surface [67]. However, the physical explanation for the origin of this elliptical morphology remains open and requires a dedicated study.

The relatively small dispersion of the $L_{x, y, z}$ values in Fig. 8 for different input pulse energies $E_{\text {in }}$ indicates that the laws of modification growth described by Eq. (2) do not depend much on this parameter in the tested input pulse energy range $\left(E_{\mathrm{in}}=1.7-2.4 \mu \mathrm{J}\right)$. This is in agreement with the saturation of the laser intensity during the propagation of ultrashort pulses in silicon [4,5]. A statistical analysis of $A_{i}$ and $B_{i}$ coefficients ( $i=x, y$, or $z$ ) appearing in Eq. (2) is recapitulated in Table I.

\section{E. Transverse inscription}

By constantly moving the sample perpendicular to the laser beam at various writing speeds $v$, transverse inscription has been attempted inside silicon at $120-\mu \mathrm{m}$ focus position (i.e., the deepest position for which $100 \%$ damage probability is reached for high $E_{\text {in }}$ values, chosen to avoid potential surface plasma production) and $E_{\text {in }}=2.5 \mu \mathrm{J}$. As shown by the overview in Fig. 9(a), our procedure allows the demonstration of contactless transverse inscription of lines in bulk silicon with femtosecond laser pulses. Finer characterizations of the line morphology in different imaging planes are displayed in Figs. 9(b) and 9(c), revealing three distinct morphologies. The first one appearing for $v=400 \mu \mathrm{m} / \mathrm{s}$ is the discrete morphology, consisting of a track of individual single-pulse modifications. This morphology associated with high writing speeds is in excellent agreement with the prediction of the phenomenological model detailed in Appendix B, which allows us to determine the threshold minimum speed value for discrete line inscription $v_{\min } \approx 262 \mu \mathrm{m} / \mathrm{s}$. In stark contrast, for $v \leqslant v_{\min }$ the lines are continuous. In particular, for $40 \leqslant v \leqslant 200 \mu \mathrm{m} / \mathrm{s}$, the line morphology is steady, i.e., with a nearly constant track width. Finally, for low speeds ( $v \leqslant 20 \mu \mathrm{m} / \mathrm{s}$ ), bulgy clusters are exhibited, and the line morphology is erratic (i.e., the track width is not constant). This complex morphology likely originates from cumulative effects as suggested in Fig. 9(c) for $v=20 \mu \mathrm{m} / \mathrm{s}$. In this case, the high number of pulses per point $(N \geqslant 10)$ associated with the slow inscription gives rise to modification growth, provoking shielding and scattering effects at the focus during the subsequent irradiations. As a consequence, the energy deposition during the subsequent irradiations is drastically reduced so that no new modification is initiated at the focus until the shielding and scattering effects stop. Finally, the evolution of the line width in the $x y$ plane is displayed in Fig. 9(d) as a function of the writing speed. The line width is the same for $v \geqslant 100 \mu \mathrm{m} / \mathrm{s}$ (i.e., $N \leqslant 2$ ) and corresponds 


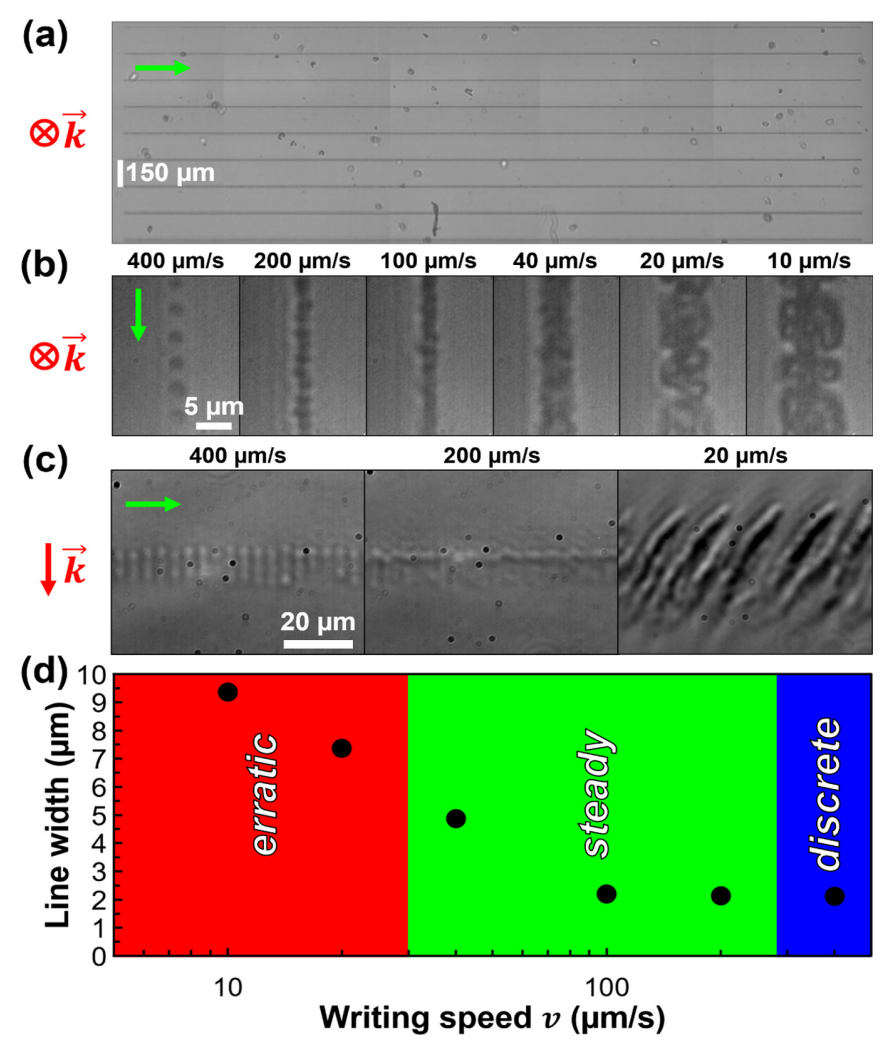

FIG. 9. Transverse ultrafast-laser-inscribed lines inside silicon. (a) Overview of 4-mm-long laser-written lines at $120-\mu \mathrm{m}$ focus position. (b) Ex situ transmission microscopy images in the $x y$ plane of lines inscribed at $E_{\text {in }}=2.5 \mu \mathrm{J}$ for different writing speeds $v$. (c) In situ transmission microscopy images in the $y z$ plane of lines inscribed at $E_{\text {in }}=2.5 \mu \mathrm{J}$ for different writing speeds $v$. In each panel, the spatial scale applies to all images, the vector $\vec{k}$ indicates the direction of laser propagation, and the green arrow shows the writing direction. (d) Evolution of the line width in the $x y$ plane as a function of $v$ for $E_{\text {in }}=2.5 \mu \mathrm{J}$. The different colors correspond to the indicated morphology domains

to the dimensions of single-pulse modifications (see Fig. 6). This demonstrates the absence of cumulative effects for high writing speeds. In contrast, for $v<100 \mu \mathrm{m} / \mathrm{s}$, the line width increases logarithmically as the writing speed decreases, thus indicating the speed for which the aforementioned cumulative effects are significant. It is noteworthy that analogous results have been obtained at $100-\mu \mathrm{m}$ focus position (not shown here), where the damage probability also reaches $100 \%$ (see Fig. 5).

Ultimately, we have studied the energy dependence of the three different morphologies exhibited in Fig. 9 (i.e., discrete, steady, and erratic) by characterizing transversely inscribed lines for various input pulse energies and writing speeds. The corresponding results are displayed in Fig. 10. As discussed previously, high input pulse energies lead to discrete and erratic morphology for excessively high and low speeds, respectively. Interestingly, for intermediate speeds, the steady morphology solely exists for $E_{\text {in }}>1.8 \mu \mathrm{J}$, corresponding to a single-pulse damage probability of $100 \%$ (see the red curve in Fig. 10). Conversely, the line morphology is discrete for $v=40-100 \mu \mathrm{m} / \mathrm{s}$ and $E_{\text {in }} \approx 1.7 \mu \mathrm{J}$, which corresponds to

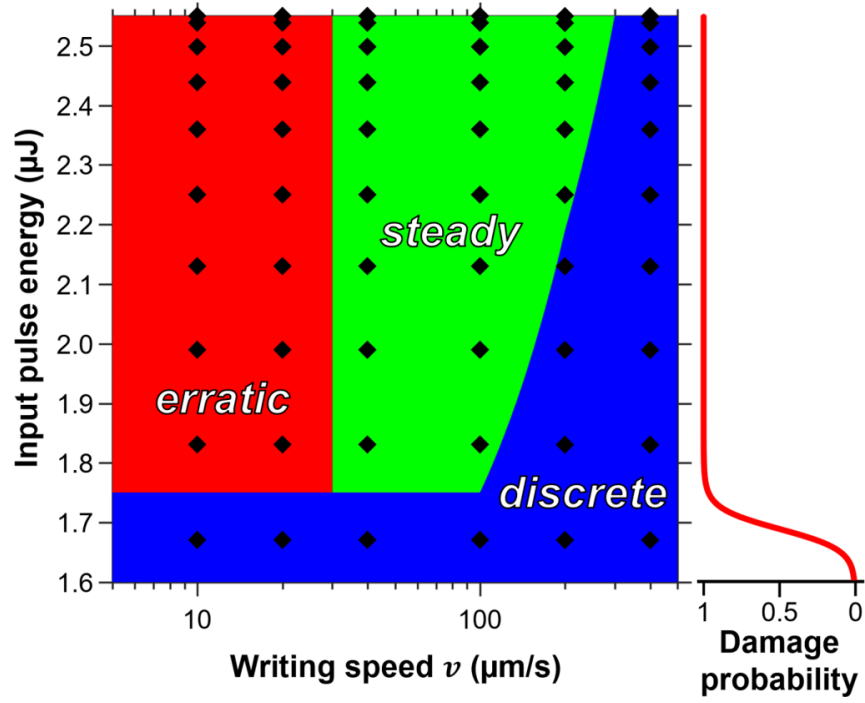

FIG. 10. Morphology domains of transversely-inscribed lines as a function of the input pulse energy and the writing speed. The focus position is $120 \mu \mathrm{m}$. The black points indicate the conditions for which line inscription has been attempted, and the color zones correspond to the different morphology domains. The red curve corresponds to a sigmoid fit of the single-pulse damage probability data in Fig. 5.

a nonzero modification probability strictly lower than $100 \%$ (see Fig. 5). Given that modification growth occurs at $E_{\text {in }} \approx$ $1.7 \mu \mathrm{J}$ (see Fig. 8), one can conclude that continuous line inscription is not related to the reignition of modifications enabling their transverse expansion, but to intrinsic permanent modification inside the material. In other words, a single-pulse modification probability of $100 \%$ is a necessary condition for the transverse inscription of continuous lines (either steady or erratic). This conclusion is all the more supported by the observations in Fig. 4.

\section{SUMMARY}

We have demonstrated the possibility to contactlessly inscribe continuous transverse lines in bulk silicon with ultrashort laser pulses. While this achievement is usually unattainable due to nonlinear propagation effects, we have proposed a procedure based on advantageous laser conditions for circumventing these limitations. This approach consists of using femtosecond pulses (i) at the beneficial wavelength of $\approx 2 \mu \mathrm{m}$, (ii) exhibiting temporal distortions, and (iii) focused at a position for which spherical aberrations of different origins mutually balance. The concomitance of these spectral, temporal, and spatial conditions was shown to be indispensable for producing repeatable permanent modifications - and thus transverse inscription-inside silicon.

Our methodology first relies on the identification of the conditions that allow one to obtain $100 \%$ damage probability with single-pulse irradiations. Deterministic damage behavior was observed for input pulse energies $E_{\text {in }} \geqslant 1.9 \mu \mathrm{J}$ and focus positions $\approx 100-120 \mu \mathrm{m}$ below the entrance surface. In this narrow focus position window, spherical aberrations of different origins mutually balance, as calculated with simple 
theoretical considerations relying on point spread function analyses. More realistic nonlinear propagation simulations showed that this mutual balance leads to enhanced energy deposition in silicon. In addition, we have studied the spatial expansion of the modifications on a pulse-to-pulse basis. By applying different numbers of pulses for various input pulse energies, we have determined the laws governing modification growth. In all spatial directions, the growth behavior was found to scale logarithmically with the number of pulses, with no strong dependence on the input pulse energy. Finally, lines have been transversely inscribed in the bulk of silicon. Depending on the writing speed and the input pulse energy, the transversely written lines may show an erratic, steady, or discrete morphology. The discrete morphology appears either for input pulse energies leading to single-pulse damage probabilities $<100 \%$ or for too high writing speeds.

The demonstration of transversely inscribed lines paves the way to achieving contactless 3D laser direct writing inside silicon. While we have concentrated our efforts on transverse line inscription in a limited range of focus positions (100$120 \mu \mathrm{m}$ ), we anticipate that phase-control elements (e.g., spatial light modulators) can be employed for precompensating spherical aberrations at arbitrary focus positions-thus allowing ultrafast laser inscription in all spatial directions. In the future, a broad range of in-chip applications can be addressed, including optical functionalization, wafer dicing, and microfluidics.

\section{ACKNOWLEDGMENTS}

This research has been supported by the Bundesministerium für Bildung und Forschung (BMBF) through the NUCLEUS project, Grant No. 03IHS107A, as well as the glass2met project, Grant No. 13N15290. It has also been supported by National Priorities Research Program Grant No. NPRP11S-1128-170042 from the Qatar National Research Fund (member of the Qatar Foundation). We acknowledge support by the German Research Foundation and the Open Access Publication Fund of the Thüringer Universitäts- und Landesbibliothek Jena, Project No. 433052568. The authors gratefully acknowledge R. Herda and A. Zach (Toptica Photonics) for technical support.

\section{APPENDIX A: CALCULATIONS OF THE DEPOSITED ENERGY}

In order to calculate the laser energy deposited to the freeelectron subsystem, we first consider the work $d W$ done by the electromagnetic field force $\vec{F}$ acting on the electrons of total charge $q$ during time interval $d t$ [68]:

$$
d W=\vec{F} \cdot \vec{d} l=q(\overrightarrow{\mathcal{E}}+\vec{v} \times \overrightarrow{\mathcal{B}}) \cdot(\vec{v} d t)=q(\overrightarrow{\mathcal{E}} \cdot \vec{v}) d t,
$$

where $\overrightarrow{\mathcal{E}}$ and $\overrightarrow{\mathcal{B}}$ are the electric and magnetic fields, respectively, and $\vec{v}$ is the electron velocity. For continuous charge distribution $q=\rho d V$, with electron density $\rho$ and volume element $d V$, we obtain

$$
\begin{aligned}
d W & =(\rho d V)(\overrightarrow{\mathcal{E}} \cdot \vec{v}) d t=\overrightarrow{\mathcal{E}} \cdot(\rho \vec{v}) d V d t \\
& =(\overrightarrow{\mathcal{E}} \cdot \vec{J}) d V d t,
\end{aligned}
$$

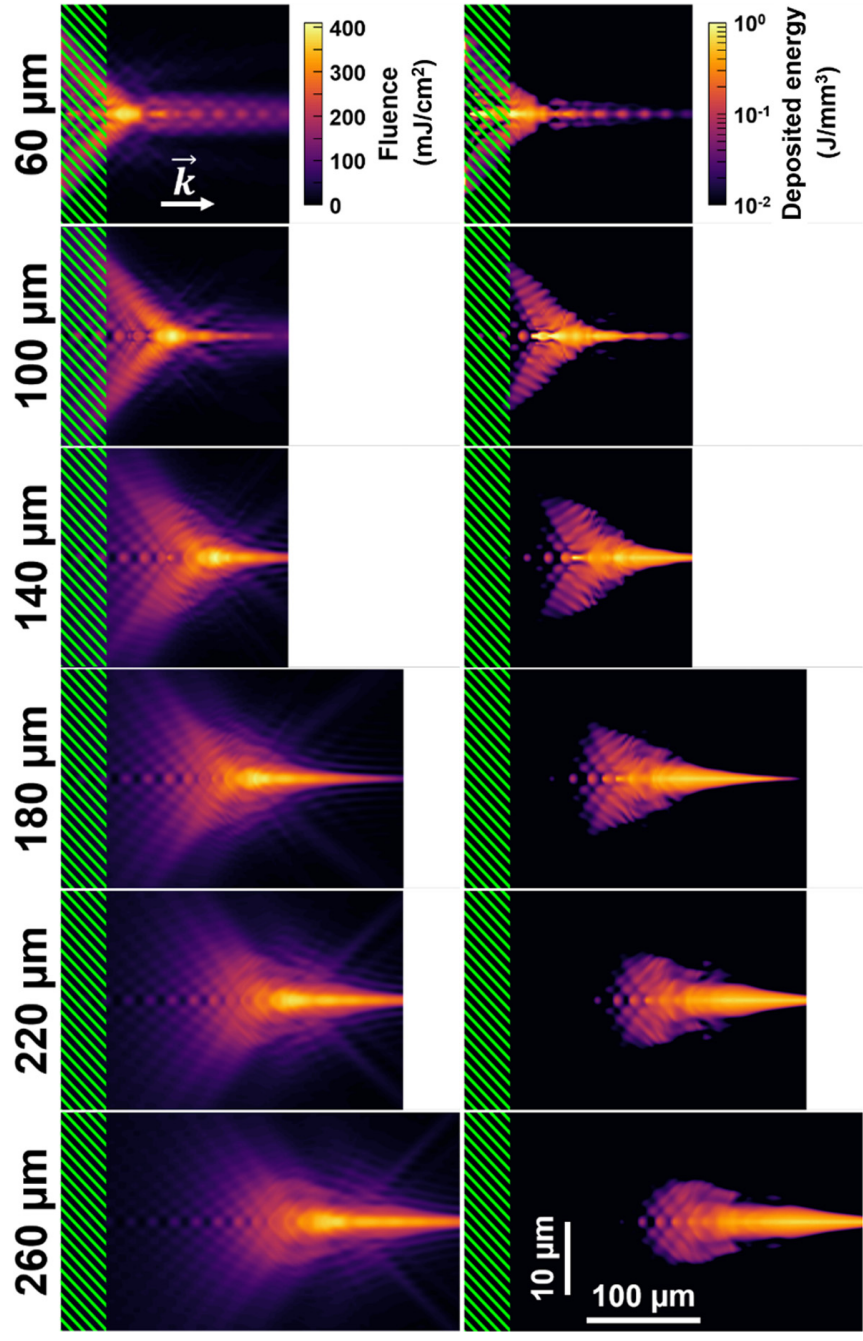

FIG. 11. Simulated fluence and deposited energy distributions for different focus positions. The green shading marks the region below $40 \mu \mathrm{m}$ corresponding to surface damage in the experiments.

where $\vec{J}=\rho \vec{v}$ is the current. Thus the work done by the electromagnetic field force per unit time, per unit volume, that is, the energy deposited to the free electrons per unit time, per unit volume, is given by

$$
\frac{d W}{d V d t}=\overrightarrow{\mathcal{E}} \cdot \vec{J}
$$

In turn, we are interested in the energy deposited per unit volume, which we calculate as

$$
\frac{d W}{d V}=\int \overrightarrow{\mathcal{E}} \cdot \vec{J} d t
$$

In our simulations we use this deposited energy, measured in units of $\mathrm{J} / \mathrm{m}^{3}$, to estimate the damage probability inside silicon [47].

In order to provide an intuition, Fig. 11 shows the fluence and deposited energy distributions, calculated by Eq. (A4), for different focus positions. One can see that, compared with the fluence, the deposited energy distribution is much more localized in space (note the logarithmic scale for the deposited energy distributions in Fig. 11). Following the experiments, 


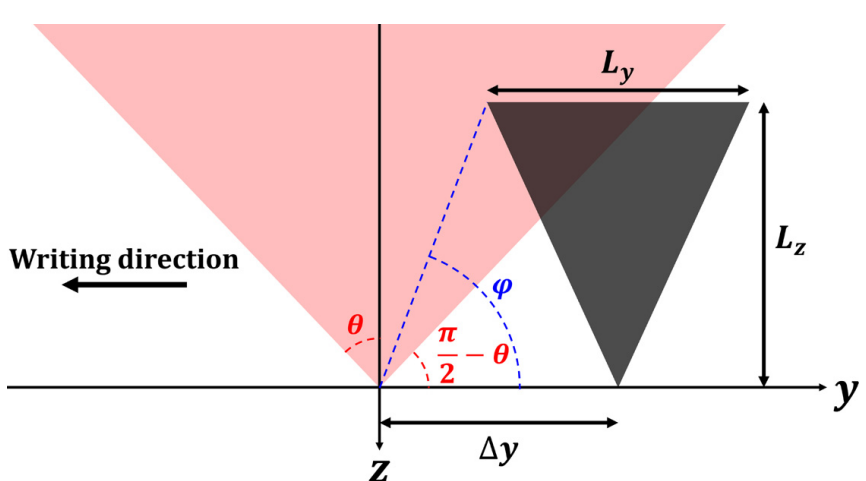

FIG. 12. Geometrical considerations associated with the phenomenological model for transverse inscription. The modification (in black) is produced by a pulse preceding the current irradiation (in pink).

we are interested only in bulk damage in silicon and therefore exclude from our analysis the energy deposited at focus positions below $40 \mu \mathrm{m}$ (marked by the green shading in Fig. 11).

\section{APPENDIX B: PHENOMENOLOGICAL MODEL FOR TRANSVERSE INSCRIPTION}

Let us examine theoretically the conditions allowing transverse inscription of continuous lines inside silicon. To do so, we develop a phenomenological model relying on the laws of modification growth established in Sec. III D and geometrical considerations. Intuitively, high writing speeds should lead to a noncontinuous (i.e., a discrete) line morphology consisting of individual single-pulse modifications next to each other. The discrete morphology thus solely exists when there is no interplay between individual modifications. In other words, the modification produced by one pulse does not provoke any shielding or scattering effect during the subsequent irradiation.

Without limiting the generality of the model, let us consider a sample movement-and thus an inscription-along the $y$ axis, as schematically depicted in Fig. 12, where the laser irradiation (in pink) is subsequent to a laser-produced modification (in black). The two consecutive irradiations are spatially separated by a distance $\Delta y=v / \Omega_{0}$, where $v$ is the writing speed and $\Omega_{0}=100 \mathrm{~Hz}$ is the laser repetition rate. In this figure, we introduce the angle $\varphi$ between the focal plane and the point of the modification with the closest distance to the optical axis $z$. The condition for discrete line inscription is thus

$$
\varphi \leqslant \frac{\pi}{2}-\theta
$$

where $\theta=\arcsin \left(\mathrm{NA} / n_{0}\right)$ is the maximum half angle of the cone of light, NA is the numerical aperture of the focusing lens, and $n_{0}$ is the refractive index of silicon at the laser wavelength. The angle $\varphi$ reads

$$
\varphi=\arctan \left(\frac{L_{z}}{\Delta y-L_{y} / 2}\right) .
$$

Here, $L_{z}$ and $L_{y}$ are the maximum lengths of the modification along $z$ and $y$, respectively. In the framework of our study, as established in Sec. IIID for modification growth at the same position, these lengths both scale logarithmically with the number of pulses as

$$
L_{y, z}=A_{y, z} \ln (N)+B_{y, z},
$$

where $A_{y, z}$ and $B_{y, z}$ are the growth coefficient and the length of a single-pulse modification along $y$ and $z$, respectively, and $N \geqslant 1$ is the number of pulses applied during the growth sequence. In the case of transverse laser inscription, where the focus position is continuously moved perpendicular to the laser, the number of pulses per point can be defined as $N=2 w_{0} \Omega_{0} / v$, where $w_{0}$ is the beam waist. Recalling that $\tan (\pi / 2-x)=1 / \tan (x)$, the combination of Eqs. (B1) and (B2) yields

$$
\frac{L_{z}}{v / \Omega_{0}-L_{y} / 2} \leqslant \frac{1}{\tan \left[\arcsin \left(\mathrm{NA} / n_{0}\right)\right]} .
$$

Finally, a condition on the writing speed $v$ can be expressed by combining Eqs. (B3) and (B4). This condition takes the form

$$
\alpha v+\beta \ln (v) \geqslant \gamma
$$

where

$$
\begin{aligned}
\alpha= & 2 / \Omega_{0}, \\
\beta= & A_{y}+2 A_{z} \tan \left[\arcsin \left(\mathrm{NA} / n_{0}\right)\right], \\
\gamma= & A_{y} \ln \left(2 w_{0} \Omega_{0}\right)+B_{y}+2\left[A_{z} \ln \left(2 w_{0} \Omega_{0}\right)\right. \\
& \left.+B_{z}\right] \tan \left[\arcsin \left(\mathrm{NA} / n_{0}\right)\right] .
\end{aligned}
$$

The threshold minimum speed $v_{\min }$ for discrete line inscription appears in Eq. (B5) when the left- and the right-hand sides are equal. In this case, the analytical solution is

$$
v_{\min }=\frac{\beta}{\alpha} W\left[\frac{\alpha}{\beta} \exp \left(\frac{\gamma}{\beta}\right)\right] .
$$

Here, $W$ denotes the Lambert $W$ function [69]. Inserting in Eq. (B6) the set of parameters determined experimentally in Sec. IIID, we find a threshold minimum speed value for discrete line inscription $v_{\min } \approx 262 \mu \mathrm{m} / \mathrm{s}$
[1] M. Beresna, M. Gecevičius, and P. G. Kazansky, Ultrafast laser direct writing and nanostructuring in transparent materials, Adv. Opt. Photonics 6, 293 (2014).

[2] M. Chambonneau, D. Grojo, O. Tokel, F. Ö. Ilday, S. Tzortzakis, and S. Nolte, In-volume laser direct writing of silicon-Challenges and opportunities, Laser Photon. Rev. 2100140 (2021), doi: 10.1002/lpor.202100140.
[3] V. V. Kononenko, V. V. Konov, and E. M. Dianov, Delocalization of femtosecond radiation in silicon, Opt. Lett. 37, 3369 (2012).

[4] V. V. Kononenko, E. V. Zavedeev, and V. M. Gololobov, The effect of light-induced plasma on propagation of intense fs laser radiation in c-Si, Appl. Phys. A: Mater. Sci. Process. 122, 293 (2016). 
[5] E. V. Zavedeev, V. V. Kononenko, and V. I. Konov, Delocalization of femtosecond laser radiation in crystalline $\mathrm{Si}$ in the mid-IR range, Laser Phys. 26, 016101 (2016).

[6] M. Chanal, V. Y. Fedorov, M. Chambonneau, R. Clady, S. Tzortzakis, and D. Grojo, Crossing the threshold of ultrafast laser writing in bulk silicon, Nat. Commun. 8, 773 (2017).

[7] E. I. Mareev, K. V. Lvov, B. V. Rumiantsev, E. A. Migal, I. D. Novikov, S. Y. Stremoukhov, and F. V. Potemkin, Effect of pulse duration on the energy delivery under nonlinear propagation of tightly focused Cr:forsterite laser radiation in bulk silicon, Laser Phys. Lett. 17, 015402 (2020).

[8] M. Chambonneau, Q. Li, V. Y. Fedorov, M. Blothe, K. Schaarschmidt, M. Lorenz, S. Tzortzakis, and S. Nolte, Taming ultrafast laser filaments for optimized semiconductor-metal welding, Laser Photonics Rev. 15, 2000433 (2021).

[9] M. Chambonneau, Q. Li, M. Chanal, N. Sanner, and D. Grojo, Writing waveguides inside monolithic crystalline silicon with nanosecond laser pulses, Opt. Lett. 41, 4875 (2016).

[10] I. Pavlov, O. Tokel, S. Pavlova, V. Kadan, G. Makey, A. Turnali, Ö. Yavuz, and F. Ö. Ilday, Femtosecond laser written waveguides deep inside silicon, Opt. Lett. 42, 3028 (2017).

[11] G. Matthäus, H. Kämmer, K. A. Lammers, C. Vetter, W. Watanabe, and S. Nolte, Inscription of silicon waveguides using picosecond pulses, Opt. Express 26, 24089 (2018).

[12] M. Chambonneau, X. Wang, X. Yu, Q. Li, D. Chaudanson, S. Lei, and D. Grojo, Positive- and negative-tone structuring of crystalline silicon by laser-assisted chemical etching, Opt. Lett. 44, 1619 (2019)

[13] H. Kämmer, G. Matthäus, K. A. Lammers, C. Vetter, M. Chambonneau, and S. Nolte, Origin of waveguiding in ultrashort pulse structured silicon, Laser Photonics Rev. 13, 1800268 (2019).

[14] A. Turnali, M. Han, and O. Tokel, Laser-written depressedcladding waveguides deep inside bulk silicon, J. Opt. Soc. Am. B 36, 966 (2019).

[15] A. Alberucci, N. Alasgarzade, M. Chambonneau, M. Blothe, H. Kämmer, G. Matthäus, C. P. Jisha, and S. Nolte, In-Depth Optical Characterization of Femtosecond-Written Waveguides in Silicon, Phys. Rev. Appl. 14, 024078 (2020).

[16] E. Ohmura, F. Fukuyo, K. Fukumitsu, and H. Morita, Modified-layer formation mechanism into silicon with permeable nanosecond laser, Int. J. Comput. Mater. Sci. Surf. Eng. 1, 677 (2007).

[17] P. C. Verburg, G. R. B. E. Römer, and A. J. Huis in 't Veld, Two-photon-induced internal modification of silicon by erbium-doped fiber laser, Opt. Express 22, 21958 (2014).

[18] O. Tokel, A. Turnalı, G. Makey, P. Elahi, T. Çolakoğlu, E. Ergeçen, Ö. Yavuz, R. Hübner, M. Zolfaghari Borra, I. Pavlov, A. Bek, R. Turan, D. K. Kesim, S. Tozburun, S. Ilday, and F. Ö. Ilday, In-chip microstructures and photonic devices fabricated by nonlinear laser lithography deep inside silicon, Nat. Photonics 11, 639 (2017).

[19] M. Chambonneau, D. Richter, S. Nolte, and D. Grojo, Inscribing diffraction gratings in bulk silicon with nanosecond laser pulses, Opt. Lett. 43, 6069 (2018).

[20] X. Wang, X. Yu, H. Shi, X. Tian, M. Chambonneau, D. Grojo, B. DePaola, M. Berg, and S. Lei, Characterization and control of laser induced modification inside silicon, J. Laser Appl. 31, 022601 (2019).

[21] X. Wang, X. Yu, M. Berg, B. DePaola, H. Shi, P. Chen, L. Xue, $X$. Chang, and S. Lei, Nanosecond laser writing of straight and curved waveguides in silicon with shaped beams, J. Laser Appl. 32, 022002 (2020).

[22] R. A. Richter, N. Tolstik, S. Rigaud, P. D. Valle, A. Erbe, P. Ebbinghaus, I. Astrauskas, V. Kalashnikov, E. Sorokin, and I. T. Sorokina, Sub-surface modifications in silicon with ultra-short pulsed lasers above $2 \mu \mathrm{m}$, J. Opt. Soc. Am. B 37, 2543 (2020).

[23] A. H. Nejadmalayeri, P. R. Herman, J. Burghoff, M. Will, S. Nolte, and A. Tünnermann, Inscription of optical waveguides in crystalline silicon by mid-infrared femtosecond laser pulses, Opt. Lett. 30, 964 (2005).

[24] V. V. P. Sreenivas, M. Bülters, and R. B. Bergmann, Microsized subsurface modification of mono-crystalline silicon via non-linear absorption, J. Eur. Opt. Soc.: Rapid Publ. 7, 12035 (2012).

[25] M. Chambonneau, L. Lavoute, D. Gaponov, V. Fedorov, A. Hideur, S. Février, S. Tzortzakis, O. Utéza, and D. Grojo, Competing Nonlinear Delocalization of Light for Laser Inscription Inside Silicon with a 2- $\mu \mathrm{m}$ Picosecond Laser, Phys. Rev. Appl. 12, 024009 (2019).

[26] A. Wang, A. Das, and D. Grojo, Temporal-contrast imperfections as drivers for ultrafast laser modifications in bulk silicon, Phys. Rev. Research 2, 033023 (2020).

[27] A. Wang, A. Das, and D. Grojo, Ultrafast laser writing deep inside silicon with THz-repetition-rate trains of pulses, Research 2020, 8149764 (2020).

[28] R. Kingslake and R. B. Johnson, Lens Design Fundamentals (Academic, New York, 2009).

[29] A. Berner, E. Kasperkiewicz, Y. Zhang, and H. Gross, 3rd-order spherochromatism surface contribution and its intrinsic and induced aberration parts, J. Opt. Soc. Am. A 35, 1368 (2018).

[30] C. Hnatovsky, R. S. Taylor, E. Simova, V. R. Bhardwaj, D. M. Rayner, and P. B. Corkum, High-resolution study of photoinduced modification in fused silica produced by a tightly focused femtosecond laser beam in the presence of aberrations, J. Appl. Phys. 98, 013517 (2005).

[31] H. H. Li, Refractive index of silicon and germanium and its wavelength and temperature derivatives, J. Phys. Chem. Ref. Data 9, 561 (1980).

[32] Q. Li, M. Chambonneau, M. Chanal, and D. Grojo, Quantitative-phase microscopy of nanosecond laser-induced micro-modifications inside silicon, Appl. Opt. 55, 9577 (2016).

[33] M. Baudisch, M. Beutler, M. Gebhardt, C. Gaida, F. Stutzki, S. Hadrich, R. Herda, K. Zawilski, P. Schunemann, A. Zach, J. Limpert, and I. Rimke, 2.3-12 $\mu \mathrm{m}$ tunable, sub-10 optical cycle, $\mathrm{ZnGeP}_{2}$-based OPA directly pumped by a Tm:fiber laser at $1.96 \mu \mathrm{m}$ and $100 \mathrm{kHz}$, in Laser Congress 2018 (ASSL) (OSA, Washington, DC, 2018), Part F121, p. AW4A.3.

[34] Q. Li, M. Chambonneau, M. Blothe, H. Gross, and S. Nolte, Flexible, fast, and benchmarked vectorial model for focused laser beams, Appl. Opt. 60, 3954 (2021).

[35] INFOCUS, 2021, https://github.com/QF06/InFocus.

[36] A. D. Bristow, N. Rotenberg, and H. M. van Driel, Two-photon absorption and Kerr coefficients of silicon for 850-2200 nm, Appl. Phys. Lett. 90, 191104 (2007). 
[37] Q. Lin, J. Zhang, G. Piredda, R. W. Boyd, P. M. Fauchet, and G. P. Agrawal, Dispersion of silicon nonlinearities in the near infrared region, Appl. Phys. Lett. 91, 021111 (2007).

[38] T. Wang, N. Venkatram, J. Gosciniak, Y. Cui, G. Qian, W. Ji, and D. T. H. Tan, Multi-photon absorption and third-order nonlinearity in silicon at mid-infrared wavelengths, Opt. Express 21, 32192 (2013).

[39] M. Kolesik and J. V. Moloney, Nonlinear optical pulse propagation simulation: From Maxwell's to unidirectional equations, Phys. Rev. E 70, 036604 (2004).

[40] V. Y. Fedorov, M. Chanal, D. Grojo, and S. Tzortzakis, Accessing Extreme Spatiotemporal Localization of High-Power Laser Radiation through Transformation Optics and Scalar Wave Equations, Phys. Rev. Lett. 117, 043902 (2016).

[41] L. Bergé, S. Skupin, R. Nuter, J. Kasparian, and J.-P. Wolf, Ultrashort filaments of light in weakly ionized, optically transparent media, Rep. Prog. Phys. 70, 1633 (2007).

[42] A. Mouskeftaras, A. V. Rode, R. Clady, M. Sentis, O. Utéza, and D. Grojo, Self-limited underdense microplasmas in bulk silicon induced by ultrashort laser pulses, Appl. Phys. Lett. 105, 191103 (2014).

[43] P. Jonsson, H. Bleichner, M. Isberg, and E. Nordlander, The ambipolar Auger coefficient: Measured temperature dependence in electron irradiated and highly injected n-type silicon, J. Appl. Phys. 81, 2256 (1997).

[44] H. Tanimura, J. Kanasaki, K. Tanimura, J. Sjakste, and N. Vast, Ultrafast relaxation dynamics of highly excited hot electrons in silicon, Phys. Rev. B 100, 035201 (2019).

[45] A. Mouskeftaras, M. Chanal, M. Chambonneau, R. Clady, O. Utéza, and D. Grojo, Direct measurement of ambipolar diffusion in bulk silicon by ultrafast infrared imaging of laserinduced microplasmas, Appl. Phys. Lett. 108, 041107 (2016).

[46] B. Chimier, O. Utéza, N. Sanner, M. Sentis, T. Itina, P. Lassonde, F. Légaré, F. Vidal, and J. C. Kieffer, Damage and ablation thresholds of fused-silica in femtosecond regime, Phys. Rev. B 84, 094104 (2011).

[47] P. A. Zhokhov and A. M. Zheltikov, Optical breakdown of solids by few-cycle laser pulses, Sci. Rep. 8, 1824 (2018).

[48] N. Bloembergen, Laser-induced electric breakdown in solids, IEEE J. Quantum Electron. 10, 375 (1974).

[49] L. B. Glebov, O. M. Efimov, G. T. Petrovskiā, and P. N. Rogovtsev, Influence of the mode composition of laser radiation on the optical breakdown of silicate glasses, Sov. J. Quantum Electron. 14, 226 (1984).

[50] A. V. Smith and B. T. Do, Bulk and surface laser damage of silica by picosecond and nanosecond pulses at $1064 \mathrm{~nm}$, Appl. Opt. 47, 4812 (2008).

[51] G. Batavičiūte, E. Pupka, V. Pyragaite, L. Smalakys, and A. Melninkaitis, Effect of longitudinal laser mode beating in damage probability measurements, in Laser-Induced Damage in Optical Materials: 2013, Proceedings of SPIE Vol. 8885 (SPIE, Bellingham, WA, 2013), p. 88851M.

[52] R. Diaz, M. Chambonneau, R. Courchinoux, P. Grua, J. Luce, J.-L. Rullier, J.-Y. Natoli, and L. Lamaignère, Influence of longitudinal mode beating on laser-induced damage in fused silica, Opt. Lett. 39, 674 (2014)

[53] L. Lamaignère, R. Diaz, M. Chambonneau, P. Grua, J.-Y. Natoli, and J.-L. Rullier, Correlation between laser-induced damage densities of fused silica and average incubation fluences at $1064 \mathrm{~nm}$ in the nanosecond regime, J. Appl. Phys. 121, 045306 (2017).

[54] M. Chambonneau, R. Diaz, P. Grua, J.-L. Rullier, G. Duchateau, J.-Y. Natoli, and L. Lamaignère, Origin of the damage ring pattern in fused silica induced by multiple longitudinal modes laser pulses, Appl. Phys. Lett. 104, 021121 (2014).

[55] M. Chambonneau, J.-L. Rullier, P. Grua, and L. Lamaignère, Wavelength dependence of the mechanisms governing the formation of nanosecond laser-induced damage in fused silica, Opt. Express 26, 21819 (2018).

[56] F. Docchio, P. Regondi, M. R. C. Capon, and J. Mellerio, Study of the temporal and spatial dynamics of plasmas induced in liquids by nanosecond Nd:YAG laser pulses 2: Plasma luminescence and shielding, Appl. Opt. 27, 3669 (1988).

[57] H. Iwata, D. Kawaguchi, and H. Saka, Crystal structures of high-pressure phases formed in $\mathrm{Si}$ by laser irradiation, Microscopy 67, 30 (2018).

[58] P. C. Verburg, L. A. Smillie, G. R. B. E. Römer, B. Haberl, J. E. Bradby, J. S. Williams, and A. J. Huis in 't Veld, Crystal structure of laser-induced subsurface modifications in Si, Appl. Phys. A: Mater. Sci. Process. 120, 683 (2015).

[59] L. Rapp, B. Haberl, C. Pickard, J. Bradby, E. Gamaly, J. Williams, and A. Rode, Experimental evidence of new tetragonal polymorphs of silicon formed through ultrafast laser-induced confined microexplosion, Nat. Commun. 6, 7555 (2015).

[60] B. Welber, C. Kim, M. Cardona, and S. Rodriguez, Dependence of the indirect energy gap of silicon on hydrostatic pressure, Solid State Commun. 17, 1021 (1975).

[61] S. Z. Karazhanov, A. Davletova, and A. Ulyashin, Straininduced modulation of band structure of silicon, J. Appl. Phys. 104, 024501 (2008).

[62] M. A. Norton, E. E. Donohue, M. D. Feit, R. P. Hackel, W. G. Hollingsworth, A. M. Rubenchik, and M. L. Spaeth, Growth of laser damage on the input surface of $\mathrm{SiO}_{2}$ at $351 \mathrm{~nm}$, in LaserInduced Damage in Optical Materials: 2006, Proceedings of SPIE Vol. 6403 (SPIE, Bellingham, WA, 2006), p. 64030L.

[63] M. Sozet, J. Neauport, E. Lavastre, N. Roquin, L. Gallais, and L. Lamaignère, Laser damage growth with picosecond pulses, Opt. Lett. 41, 2342 (2016).

[64] M. Chambonneau and L. Lamaignère, Multi-wavelength growth of nanosecond laser-induced surface damage on fused silica gratings, Sci. Rep. 8, 891 (2018).

[65] M. Mori, Y. Shimotsuma, T. Sei, M. Sakakura, K. Miura, and H. Udono, Tailoring thermoelectric properties of nanostructured crystal silicon fabricated by infrared femtosecond laser direct writing, Phys. Status Solidi A 212, 715 (2015).

[66] A. Das, A. Wang, O. Uteza, and D. Grojo, Pulse-duration dependence of laser-induced modifications inside silicon, Opt. Express 28, 26623 (2020).

[67] K. P. Luong, R. Tanabe-Yamagishi, N. Yamada, and Y. Ito, Micromachining of the back surface and interior of Si using infrared femtosecond laser pulses, J. Laser Appl. 32, 012017 (2020).

[68] D. J. Griffiths, Introduction to Electrodynamics, 4th ed. (Pearson Education, Boston, 2013), Chap. 8, p. 357.

[69] R. M. Corless, G. H. Gonnet, D. E. G. Hare, D. J. Jeffrey, and D. E. Knuth, On the Lambert $W$ function, Adv. Comput. Math. 5, 329 (1996). 